\title{
Correction to: Informing antimicrobial management in the context of COVID-19: understanding the longitudinal dynamics of C-reactive protein and procalcitonin
}

Damien K. Ming ${ }^{1,2^{*}}$ (D), Ashleigh C. Myall ${ }^{2,6}$, Bernard Hernandez ${ }^{2}$, Andrea Y. Weiße ${ }^{3,4}$, Robert L. Peach ${ }^{5,6}$, Mauricio Barahona ${ }^{6}$, Timothy M. Rawson ${ }^{1,2}$ and Alison H. Holmes ${ }^{1,2^{*}}$

\section{Correction to: BMC Infect Dis (2021) 21:932}

https://doi.org/10.1186/s12879-021-06621-7

Following publication of the original article [1], the authors identified an error in the author name of Bernard Hernandez.

The incorrect author's name is: Bernarnd Hernandez;

The correct author's name is: Bernard Hernandez;

The author group has been updated above and the original article [1] has been corrected.

\section{Author details}

${ }^{1}$ Centre for Antimicrobial Optimisation, Hammersmith Hospital, Imperial College London, Du Cane Road, London W12 ONN, UK. ${ }^{2}$ National Institute for Health Research Health Protection Research Unit in Healthcare Associated Infections and Antimicrobial Resistance, Imperial College London,
Hammersmith Campus, Du Cane Road, London W12 0NN, UK. ${ }^{3}$ School of Informatics, University of Edinburgh, Scotland, UK. ${ }^{4}$ School of Biological Science, University of Edinburgh, Scotland, UK. ${ }^{5}$ Department of Neurology, University Hospital of Würzburg, 97080 Würzburg, Germany. ${ }^{6}$ Department of Mathematics, Imperial College London, London, UK.

Published online: 21 September 2021

Reference

1. Ming DK, Myall AC, Hernandez B, Weiße AY, Peach RL, Barahona M, Rawson TM, Holmes AH. BMC Infect Dis. 2021;21:932. https://doi.org/10.1186/ s12879-021-06621-7.

\section{Publisher's Note}

Springer Nature remains neutral with regard to jurisdictional claims in published maps and institutional affiliations. 